\title{
Fabrication and magnetic properties of hard/soft magnetostatically coupled FePt/FeNi multilayer microwires
}

\author{
J. Torrejón, ${ }^{\text {a) }}$ G. Badini-Confalonieri, and M. Vázquez \\ Institute of Materials Science, CSIC, 28049 Madrid, Spain
}

(Presented on 6 November 2007; received 10 September 2007; accepted 11 October 2007; published online 24 January 2008)

\begin{abstract}
A family of multilayer microwires with hard/soft biphase magnetic behavior is here introduced. The microwires consist of a $\mathrm{Fe}_{63} \mathrm{Pt}_{27} \mathrm{Si}_{10}$ hard magnetic nucleus and a $\mathrm{Fe}_{20} \mathrm{Ni}_{80}$ soft outer shell separated by an intermediate insulating Pyrex glass microtube. The precursor FePtSi glass-coated microwire is fabricated by quenching and drawing technique, and its $L 1_{0}$ hard magnetic phase is grown by postannealing treatment technique. The polycrystalline FeNi soft magnetic outer shell has been deposited by electroplating. The analysis of the low-field hysteresis loops of the FeNi soft phase after premagnetizing until near magnetic saturation provides information about the magnetostatic coupling between phases. The FeNi magnetization curve is shifted toward positive field when the $\mathrm{FePt}$ remanent magnetization is positive and vice versa. A systematic analysis of the magnetostatic coupling and the corresponding bias field arising from uncompensated poles of the premagnetized FePt hard phase has been performed. The strength of the bias field is shown to increase with the reduction of thickness of the FeNi layer. These magnetostatically coupled biphase systems are thought to be of large potential interest as sensing elements in sensor devices. (C) 2008 American Institute of Physics. [DOI: 10.1063/1.2830537]
\end{abstract}

\section{INTRODUCTION}

The study of multilayer magnetic systems is of large interest due to their high potential in magnetic recording media and sensor device technologies. The magnetic behavior of these systems can be tailored by the magnetic coupling between different layers which can have different origins: (i) exchange coupling between ferro/ferromagnetic or ferro/ antiferromagnetic nanometer interfaces, with a number of applications in spintronic devices, ${ }^{1}$ (ii) interlayer exchange coupling of indirect Ruderman-Kittel-Kasuya-Yosida-like interaction in ferromagnetic and nonmagnetic metallic multilayers, suitable for applications in recording and reading heads, ${ }^{2}$ and (iii) long-range dipolar coupling. In the latter case, two mechanisms can be claimed to be at the origin of the dipolar bias: orange peel (Néel) magnetostatic coupling in the presence of a correlated roughness at both spacer interfaces ${ }^{3}$ and magnetostic coupling through stray fields.

Several studies on the magnetostatic coupling through stray fields in interacting magnetic bilayers have been previously carried out. For example, a system with magnetostatic coupling is more useful with respect to uncoupled one in order to design Magnetoresistive Random Access Memory (MRAM) devices with smaller writing time. ${ }^{4}$ On the other hand, in CoPt squares embedded into a Permalloy matrix, the magnetostatic interaction induces a long range ordered domain pattern. ${ }^{5}$ Moreover, the effect of domain replication in $\mathrm{CoPt}$ interacting bilayers is ascribed to magnetostatic coupling. ${ }^{6}$

Recently, micrometric biphase systems consisting of a soft nucleus and a magnetically harder outer layer have been

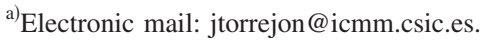

introduced. $^{7}$ The inner nucleus is a glass-coated microwire obtained by quenching and drawing technique which properties have been recently reviewed. ${ }^{8}$ The outer magnetic layer is prepared by electrochemical route. ${ }^{9}$ Bias effects in these systems have been confirmed to arise from uncompensated magnetic charges at the hard shell. ${ }^{10}$ They have been also proposed as sensing elements in multifunctional sensor devices. ${ }^{11}$

In the present work, we introduce a novel family of biphase microwires where the nucleus is the hard phase, in opposition to previous mentioned reports, and the outer layer exhibits a softer magnetic behavior. We consider a FePt base alloy as hard nucleus and a FeNi soft alloy as outer layer. Besides the details about the preparation and structure characterization of the samples, the magnetic characterization is presented in the next sections.

\section{EXPERIMENTAL DETAILS AND RESULTS}

A glass-coated microwire composed by ferromagnetic nucleus with nominal composition $\mathrm{Fe}_{63} \mathrm{Pt}_{27} \mathrm{Si}_{10}, 40 \mu \mathrm{m}$ in diameter, surrounded by an insulating glass layer, $10 \mu \mathrm{m}$ thick, has been fabricated by means of quenching and drawing method. ${ }^{12}$ Suitable heat treatment produces an abrupt magnetic hardening. ${ }^{13,14}$ In this study, conventional furnace heating in regular atmosphere over a temperature range from 400 to $1000 \mathrm{~K}$ was used. In order to avoid interaction with an external magnetic field, treatments were performed in a nonmagnetic tubular furnace while the glass layer of microwire was protecting the core from oxidation.

The magnetic properties of the FePt glass-coated microwires for different heat treatments have been studied with a vibrating sample magnetometer. Figure 1(a) shows the hysteresis loops of a FePt glass-coated microwire before and 

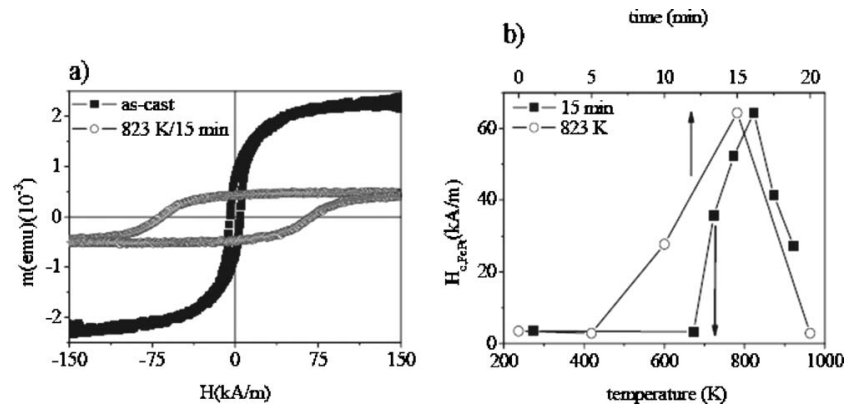

FIG. 1. (a) Hysteresis loops of FePtSi glass-coated microwire before and after heat treatment at $823 \mathrm{~K}$ for $15 \mathrm{~min}$. (b) Dependence of coercivity on temperature and time of heat treatment.

after annealing at $823 \mathrm{~K}$ for $15 \mathrm{~min}$. The heat treatment gives rise to a magnetically harder phase with reduced saturation magnetic moment. ${ }^{15}$ The dependence of the coercive field $\left(H_{c, \mathrm{FePt}}\right)$ on isochronal and isothermal treatments is shown in Fig. 1(b). Maximum coercivity of $64 \mathrm{kA} / \mathrm{m}$ is reached after annealing at $823 \mathrm{~K}$ for $15 \mathrm{~min}$.

The magnetic hardening of the FePt alloy should be ascribed to the generation of a new crystalline phase. X-ray analysis of samples before and after annealing has been performed. Previous to the $\mathrm{x}$-ray analysis, the glass cover has been removed using a fluorhydric acid solution. Figure 2 shows the corresponding x-ray spectra. For the soft sample (as-cast FePt), a fcc crystalline structure, disordered phase, $L 1_{2}$, is detected. After the heat treatment at $823 \mathrm{~K}$ and 15 min, a change of phase is produced, obtaining a fct ordered, $L 1_{0}$, corresponding to the hard magnetic phase. This agrees with previous reports. ${ }^{15-17}$

The magnetic outer shell is electroplated onto a tiny $\mathrm{Au}$ nanolayer previously sputtered onto the precursor glass coated FePt microwire. The process has been described in detail elsewhere. ${ }^{7,9}$ The FeNi shell electroplating has been done in an aqueous solution of $\mathrm{FeSO}_{4} \cdot 7 \mathrm{H}_{2} \mathrm{O}(8 \mathrm{~g} / 1)$, $\mathrm{NiSO}_{4} \cdot 6 \mathrm{H} 2 \mathrm{O} \quad(125 \mathrm{~g} / 1), \quad \mathrm{NiCl}_{2} \cdot 6 \mathrm{H}_{2} \mathrm{O}(20 \mathrm{~g} / 1), \mathrm{H}_{3} \mathrm{BO}_{3}$ $(40 \mathrm{~g} / 1)$, and saccharine $(6 \mathrm{~g} / 1) .1 M \mathrm{KOH}$ has been added to adjust the solution $p \mathrm{H}$ to $2.8{ }^{18}$ Electroplating has been carried out under a constant current density regime at $328 \mathrm{~K}$ with stirring. The analysis of FeNi composition has been performed by means of total reflection x-ray fluorescence as a function of electroplating parameters. For a wide range of time and current density of deposition, the FeNi alloy com-

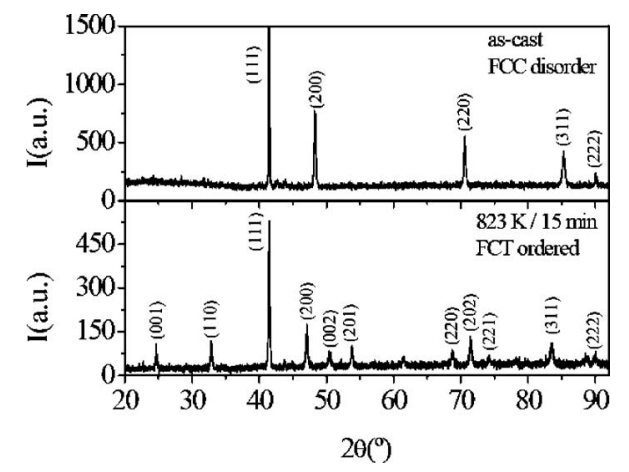

FIG. 2. X-ray spectra of FePtSi microwires before and after heat treatment at $823 \mathrm{~K}$ for $15 \mathrm{~min}$.
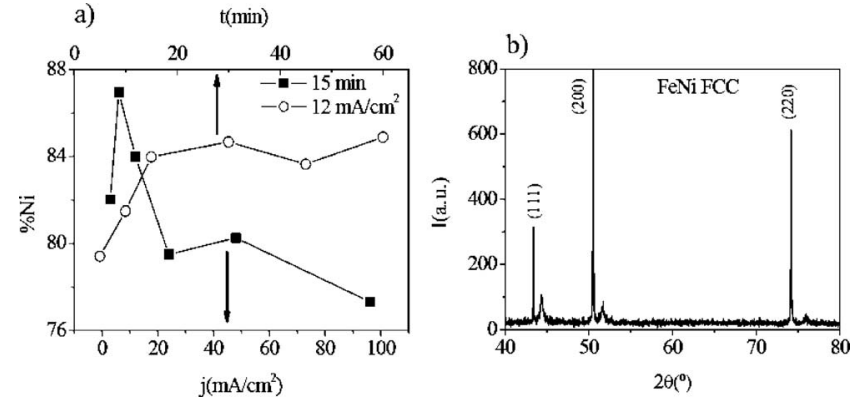

FIG. 3. (a) Ni content as a function of current density ( $\square$ ) and time $(\bigcirc)$ of electroplating and (b) x-ray spectrum of FeNi soft magnetic layer.

position is close to the Permalloy composition, $\mathrm{Fe}_{20} \mathrm{Ni}_{80}$, as shown Fig. 3(a). The FeNi thickness is proportional to time and current density of electroplating. The plated FeNi presents a fcc crystalline structure as shown in the x-ray spectrum of Fig. 3(b).

The high-field hysteresis loops for multilayer microwires, FePt nucleus, and FeNi outer shell, with different FeNi thicknesses $\left(t_{\mathrm{FeNi}}\right)$ are characterized by two steps denoting its magnetic biphase behavior, as observed in Fig. 4(a): (i) a sharp Barkhausen jump at low field (of the order of $100 \mathrm{~A} / \mathrm{m}$ ) corresponding to the magnetization reversal of the FeNi soft outer shell, and (ii) a less steeped jump at higher field (around $60 \mathrm{kA} / \mathrm{m}$ ), ascribed to the magnetization reversal of the FePt hard nucleus. An increasing of the soft phase volume with $t_{\mathrm{FeNi}}$ can be observed. In fact, the coercivity of the biphase system, $H_{c}$, is mainly determined by the coercivity of the FePt hard nucleus, $\mathrm{H}_{c \text {, FePt }}$, for smaller values of $t_{\mathrm{FeNi}}$, and by the FeNi soft outer shell, $H_{c, \mathrm{FeNi}}$, for larger values as shown in Fig. 4(b). Individual coercivity values of FePt hard layer and FeNi soft outer shell are also indicated (dashed lines).

From the study of the hysteresis loops at high field, we cannot extract direct information about the magnetic interaction between two magnetic phases. The analysis of the magnetic coupling should be performed at low-field measurements under the action of the premagnetized hard phase. In the low-field region, the hard phase magnetization remains practically constant at its remanent state, and only the reversal magnetization process of the soft phase contributes to the hysteresis loop. The low-field hysteresis loops of a multilayer microwire, $t_{\mathrm{FeNi}}=2 \mu \mathrm{m}$, with the hard phase premagnetized at $\pm 800 \mathrm{kA} / \mathrm{m}$ is plotted in Fig. 5 (a). It is observed that the hysteresis loop of the soft outer shell is
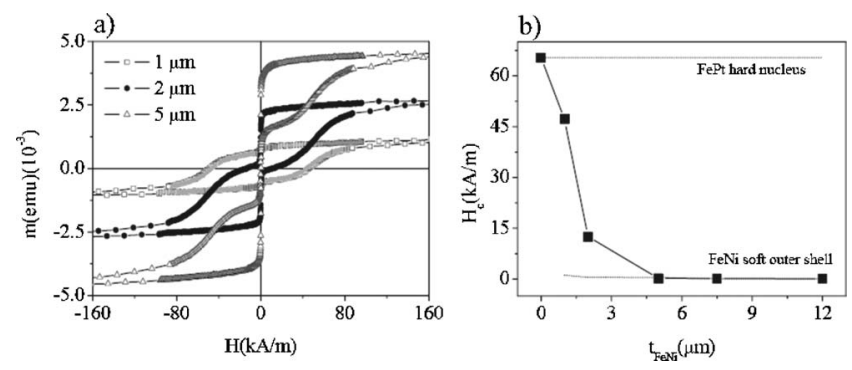

FIG. 4. (a) High-field hysteresis loops and (b) dependence of coercivity for multilayer microwires with different FeNi thicknesses. 

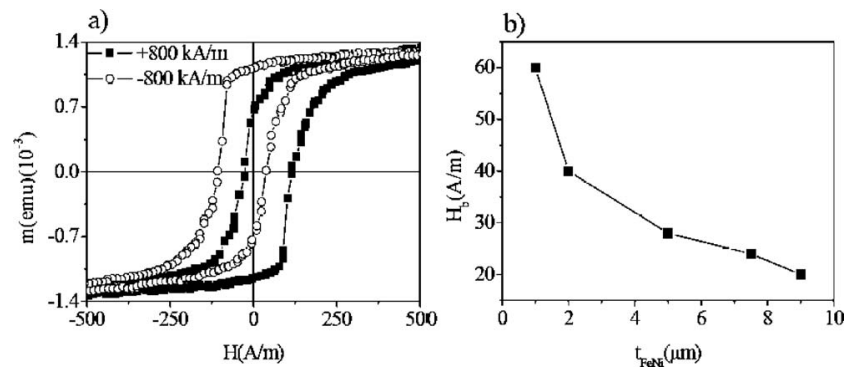

FIG. 5. (a) Low-field hysteresis loops of multilayer microwire, $t_{\mathrm{FeN}}$ $=2 \mu \mathrm{m}$, premagnetized at $\pm 800 \mathrm{kA} / \mathrm{m}$ and (b) dependence of bias field on FeNi thickness.

shifted toward positive field when the hard phase is premagnetized at $+800 \mathrm{kA} / \mathrm{m}$ and vice versa. Similar behavior has already been observed in other biphase systems. ${ }^{10,19,20}$

The origin of this shift can be explained as follows: after the hard phase is premagnetized at high field, uncompensated charges remain at the remanence state mostly at its ends. Such magnetic charges create an external field, labeled bias field $\left(H_{b}\right)$, shifting the low-field reversal magnetization process of the soft outer shell. Then, biphase system presents magnetostatic coupling through the stray field of the hard phase. The bias field is opposite to the premagnetizing field, and to the magnetization at the hard nucleus, and therefore the magnetostatic interaction presents "antiferromagneticlike" character. ${ }^{10,19,20}$

Due to the magnetostatic character of the coupling, the dimensions of the different layers play a very important role. ${ }^{20}$ So, we have performed a first study of evolution of the magnetostatic coupling with the thickness of the soft outer shell, $t_{\mathrm{FeNi}}$. Figure 5(b) shows the decrease of $H_{b}$ with $t_{\mathrm{FeNi}}$. The bias field, demagnetizing field of the hard phase, is not homogeneous, being stronger near the ends of the microwire system where uncompensated charges from the hard layer accumulate. ${ }^{21}$ As FeNi thickness increases, the bias field inside this layer becomes less intense, and therefore the shift of the magnetization curve is reduced. The study with the thickness of the hard phase has not been performed because of the technical difficulty to obtain quenched and drawn FePt glass-coated microwires with different nucleus diameter and the same glass thickness. The domain structure and magnetic behavior of the FePt are determined mainly by internal stresses induced during the solidification process. However, in biphase systems consisting of opposite magnetic configuration (soft nucleus and hard outer shell), an increase of the bias field with the hard phase thickness has been reported. ${ }^{10,19,20}$

Concerning the influence of the microwire length, it has to be restricted to a maximum length of $7 \mathrm{~mm}$ as determined by the vibrating sample magnetometer requirements. We expect an increase of the bias field with the reduction of the length as a consequence of the increase of demagnetization field, as reported for other biphase systems. ${ }^{19,20}$
In conclusion, we have introduced a family of multilayer microwire with biphase magnetic behavior. The microwire is composed by a FePt hard magnetic nucleus with fct ordered structure, an intermediate glass layer, and an FeNi soft magnetic outer shell with fcc structure. The biphase system presents magnetostatic coupling which is ascribed mainly to uncompensated poles at the ends of the premagnetized hard phase. This demagnetizing field produces a bias field that shifts the soft phase magnetization toward the orientation of premagnetizing field. Due to the magnetostatic character of the interaction between magnetic layers, the relative geometry dimensions of the layers play a very important role. In fact, the bias field decrease when the FeNi thickness increases.

\section{ACKNOWLEDGMENTS}

The work has been developed under the Project No. MAT2004-00150 supported by the Spanish Ministry of Education and Science, MEC. J. T. acknowledges a FPU program fellowship from MEC.

${ }^{1}$ J. Nogués, J. Sort, V. Langlais, V. Skumryev, S. Suriñach, J. S. Muñoz, and M. D. Baró, Phys. Rep. 422, 65 (2005).

${ }^{2}$ M. N. Baibich, J. M. Broto, A. Fert, F. Nguyen Van Dau, F. Petroff, P. Etienne, G. Creuzet, A. Friederich, and J. Chazelas, Phys. Rev. Lett. 61, 2472 (1988)

${ }^{3}$ L. Néel, C. R. Hebd. Seances Acad. Sci. 255, 1676 (1962).

${ }^{4}$ M. Carpentieri, G. Finochio, B. Azzerboni, L. Torres, E. Martínez, and L. López-Díaz, Mater. Sci. Eng., B 126, 190 (2006).

${ }^{5}$ S. Schnittger, S. Dreyer, Ch. Jooss, S. Sievers, and U. Siegner, Appl. Phys. Lett. 90, 042506 (2007).

${ }^{6}$ V. Baltz, A. Marty, B. Rodmacq, and B. Dieny, Phys. Rev. B 75, 014406 (2007).

${ }^{7}$ K. Pirota, M. Provencio, K. García, P. Mendoza, M. Hernandez-Velez, and M. Vázquez, J. Magn. Magn. Mater. 290, 68 (2005).

${ }^{8}$ M. Vázquez, in Handbook of Magnetism and Advanced Magnetic Materi$a l s$, edited by H. Kronmüller and S. Parkin (Wiley, Chichester, UK, 2007), pp. 2193-2226.

${ }^{9}$ K. Pirota, M. Hernández-Vélez, D. Navas, A. Zhukov, and M. Vázquez, Adv. Funct. Mater. 14, 266 (2004).

${ }^{10}$ J. Torrejón, G. Badini, K. Pirota, and M. Vázquez, Acta Mater. 55, 4271 (2007).

${ }^{11}$ M. Vázquez, K. Pirota, G. Badini, J. Torrejón, and H. Pfützner, European Patent No. PCT/ES2005/070173 (11, 11, 2006).

${ }^{12}$ A. Zhukov, J. González, M. Vázquez, V. Larin, and A. Torcunov, in Encyclopedia of Nanoscience and Nanotechnology, edited by H. S. Nalwa (American Scientific, Stevenson Ranch, CA, 2004), Chapter 62, Vol. 6, pp. 365-367.

${ }^{13}$ S. Shimizu and K. Watai, J. Jpn. Inst. Met. 29, 822 (1965).

${ }^{14}$ R. M. Bozorth, Ferromagnetism (Van Nostrand, New York, 1951).

${ }^{15}$ A. Kussman and G. V. Rittberg, Z. Metallkd. 42, 470 (1950).

${ }^{16}$ L. Graf and A. Kussman, Z. Phys. 36, 544 (1935).

${ }^{17} \mathrm{H}$. Lipson, D. Schoenburg, and G. V. Stupart, J. Inst. Met. 67, 333 (1941).

${ }^{18}$ X. P. Li, Z. J. Zhao, H. L. Seet, W. M. Heng, T. B. Oh, and J. Y. Lee, J. Appl. Phys. 94, 6655 (2003).

${ }^{19}$ L. Kraus, K. R. Pirota, J. Torrejón, and M. Vázquez, J. Appl. Phys. 101, 063910 (2007).

${ }^{20}$ J. Torrejón, L. Kraus, K. R. Pirota, G. Badini, and M. Vázquez, J. Appl. Phys. 101, 09N105 (2007)

${ }^{21}$ J. Torrejón, L. Kraus, G. Badini-Confalonieri, and M. Vázquez, Acta Mater. 56, 292 (2008). 